\title{
Hans Jacob Mumenthaler (1729-1813), ein bernischer Opticus und Mechanicus
}

\author{
Von E. Hintzsche, Bern
}

Auf den Namen Jacoв Mumenthaler bin ich zuerst in der Geschichte Berns von Richard Feller (Band 3, S. 551) gestoßen. Er berichtet in dem betreffenden Kapitel, daß die handwerklichen Berufe in Bern während des 18. Jahrhunderts wenig geachtet waren und daher im ganzen Kanton eher zurückgingen. Immerhin schloß Feller den Abschnitt mit einigen tröstlichen Sätzen, daß es nämlich auch damals «dem Berner Land nicht an geschickten Händen und erfinderischen Köpfen » gefehlt hätte; unter den als Beleg dafür Genannten steht neben anderen Jacob Mumenthaler von Langenthal, von dem es heißt, er «bildete sich selbst zum Optiker und verfertigte Fernrohre und Mikroskope».

Einem historisch interessierten Anatomen, der nicht nur den Forschungsergebnissen, sondern auch den technischen Belangen seines Berufes Aufmerksamkeit schenkt, wird ein solcher Hinweis natürlich zur Verpflichtung, diesem Manne nachzugehen. Die größeren Werke über die Geschichte der Mikroskope, wie das von Harting (1859) und das von Clay and Court (1932), nennen Jacob Mumenthaler nicht, doch ist er in der neueren Literatur - wenigstens der schweizerischen - nicht völlig vergessen. So wird der einzige zur Zeit bekannte Brief Jacob Mumenthalers wörtlich in dem von Eugen Kohler (1932) herausgegebenen Werk Alt-Langenthal in Wort und Bild mitgeteilt; in anderem Zusammenhang wird darüber zu berichten sein. Ferner schrieb H. Tribolet (1929) im Historisch-Biographischen Lexikon der Schweiz (Band 5, S. 207) eine kurze Notiz über Jacob Mumenthaler: Er sei zuerst Buchbinder gewesen, hätte sich als Autodidakt dem Studium der Physik gewidmet und beinahe ohne fremde Anleitung eine Menge optischer Instrumente, Fernrohre, einfache und zusammengesetzte Mikroskope usw. angefertigt, die 1773 von der Akademie der Wissenschaften in Paris geprüft und ausgezeichnet worden seien.

Diese Angaben sind jedoch ganz offensichtlich nur älteren Quellen entnommen. Geht man diesen nach, so wird man zunächst die fünfbändige Sammlung bernischer Biographien einsehen, die 1880-1906 im Auftrage des Historischen Vereins des Kantons Bern veröffentlicht wurde, leider aber unvollständig blieb. Mit vielen anderen wird Jacob Mumenthaler dort nur un- 
ter denen genannt, deren Lebenslauf eigentlich noch hätte bearbeitet werden müssen (5. Band, S. 7, Spalte 1, und S. 637, Spalte 3).

Dem freundlichen Entgegenkommen eines Fakultätskollegen des Neurologen Professor M. Mumenthaler in Bern verdanke ich die Möglichkeit, Einsicht in das Familienbuch der Mumenthaler nehmen zu können. Leider sind darin nur die Familientafeln einiger Linien der Träger dieses Namens zusammengestellt; über den alten Opticus und Mechanicus fand sich darin nichts. Dagegen konnte ich einige personelle Angaben aus alten Kirchenbüchern durch das Zivilstandsamt Langenthal erhalten. Danach ist Hans Jacob Mumenthaler am 21. August 1729 in Langenthal getauft worden, das Geburtsdatum ist, wie so häufig in jener Zeit, nicht eingetragen; sein Vater hieß Jacob Mumenthaler, die Mutter Anna Maria Dennler. Geheiratet hat Hans Jacob Mumenthaler am 19. Februar 1762, also im Alter von 32 Jahren; seine Frau Elsbeth Christen war von Thörigen. Zwei Knaben, die in den ersten Ehejahren geboren wurden, starben schon bald. Über eventuelle weitere Kinder und von diesen abzuleitende direkte Nachkommen weiß ich nichts, man müßte dazu die Tauflisten noch genauer durchsehen. Gestorben ist der «Opticus» Jacob Mumenthaler im Alter von 84 Jahren am 7. März 1813.

Diese Akten geben also nur gerade das Gerüst des Lebenslaufes, sie verhelfen aber noch nicht zu Kenntnissen über die Tätigkeit und die Leistungen Jacob Mumenthalers. Für solche muß man schon ziemlich weit zurückgehen, bis ins Jahr 1826, in dem MARKus Lutz den 2. Band seiner Sammlung Moderne Biographien herausgab; sie betreffen insbesondere interessante Männer der damaligen Zeit, die sich in verschiedenen Berufen ..., so auch als Gelehrte und Künstler in der Schweiz ausgezeichnet hatten. Lutz schrieb (S. 230-232): «Mumenthaler, (Jacob,) Optiker und Mitstifter der helvetisch-naturforschenden Gesellschaft, geb. zu Langenthal 1729. Sein Vater war Spezereyhändler, und wollte den Sohn dem gleichen Gewerbe wiedmen, weil aber dieser wenig Neigung zu demselben äußerte, überließ er es, als einsichtsvoller Mann, dem Sohne selbst, sich ein Fach zu wählen, und der Jüngling, der großes Vergnügen an der Lektüre fand, wählte den Buchbinderberuf für seine künftige Bestimmung. Sich in demselben zu vervollkommnen, begab er sich auf Reisen, durchwanderte einen Theil von Frankreich und Deutschland, und arbeitete in einigen vorzüglichern Städten dieser Länder. Diese Wanderschaft hatte aber auch auf seine spätere wissenschaftliche Bildung einen sehr vorteilhaften Einfluß, da er ausgezeichnete Anlagen zu physischen [physikalischen] Kenntnissen und Arbeiten besaß, 
in welchen er sich, ohne die seines erlernten Handwerks bey Seite zu setzen, übte. Nachdem er sich in seinem Vaterort niedergelassen hatte, folgte er seinem überwiegenden Hange zur Physik noch mehr, durchlas alle auf dieselbe Bezug habenden Bücher und Schriften, die er auftreiben konnte, und erwarb sich durch seinen unermüdeten Eifer und Forschungsgeist solche tiefe und ausgebreitete Einsichten in alle Theile dieser Wissenschaft und ihrer Hülfsmittel, daß sein Name, als eines wahren Autodidactus, nicht lange verborgen blieb. Er verfertigte z. B. einen vollständigen elektrischen Apparat, mit dem er mehrere schöne Versuche anstellte, auch bisher unbekannte, und vor ihm unerprobte Experimente vollbrachte, und in der Optik hatte er solche Fortschritte gemacht, daß er Fernrohre, Teleskope, Mikroskope, einfach und zusammengesetzt, Solar-Mikroskope usw. nicht nur durch eigenen Fleiß zu der größtmöglichen Vollkommenheit brachte und sie liefern konnte, sondern er verbesserte auch die Letztern vermittelst einer von ihm erfundenen Vorrichtung, um sie sogar bey undurchsichtigen Objekten anwenden zu können. Mit einem Dutzend dieser auf das Geschmackvollste bearbeiteten optischen Instrumente reiste Mumenthaler im J. 1773 nach Paris, wo die Akademie der Wissenschaften dieselben prüfte und mit ihrem Beyfall beehrte, diesen auch in der Wochenschrift: Avant-Coureur, daselbst aussprach. Nach seiner Rückkehr nach Hause entsagte er hierauf seinem Brodberufe, um nicht mehr die Stunden borgen zu müssen, welche er seinem Lieblingsstudium wiedmen wollte, und der erfahrene und geschickte Physiker verdrängte nun bey ihm den wohlbewanderten Buchbinder. Mit schöpferischem Auge durchlief er von jetzt an den großen weiten Kreis der Optik, so wie der Mechanik, studierte rastlos Alles, was die Literatur hierüber zu Tage förderte, wodurch er auch seinem Geiste die völlige Reife gab, deren er fähig war. Seine verfertigten Arbeiten, unter welchen Teleskope von 50 Louis d'ors am Werthe waren, gewannen ihm viele und große Gönner und Freunde, die in ihm ein Genie bewunderten, das ohne fremde Beyhülfe den Pfad des Ruhmes betreten, und sich emporgeschwungen hatte. Im J. 1797 hatte sich Mumenthaler mit mehreren zum Theil noch lebenden würdigen Naturforschern von Bern und Genf zu einer helvetisch-naturforschenden Gesellschaft vereinigt, die ihre erste Versammlung im Oktober gleichen Jahres in Herzogenbuchsee feyerte, aber durch die bald darauf erfolgte Revolution wieder in's Stocken gerieth, von welcher jedoch der gegenwärtig blühende Verein naturforschender Freunde in der Schweiz sein rühmliches Daseyn ableitet. Mumenthalers Fähigkeiten nahmen selbst mit den Jahren nicht ab, und was er über Blitzableitung, Elektrizität und Optik 
noch in seinen späteren Tagen geschrieben hat, obgleich seine hinterlassenen Manuskripte die Presse nicht gesehen haben, ist hievon ein unverwerflicher Zeuge. Er starb im Jahre 1813, beynahe 85 Jahre alt, im Genuße einer liebevollen Achtung aller derer, die ihn kannten, und welche ein durch Denken und Wissen geläutertes, und für die Ewigkeit veredeltes Gemüth verdient.»

Einige Punkte aus dieser Biographie möchte ich als wesentlich hervorheben. Richtig ist z. B. seine Mitwirkung bei der ersten Gründung einer «Allgemein helvetischen Gesellschaft der Freunde der vaterländischen Physik und Naturgeschichte». Daß er von den übrigen 12 Wissenschaftern zu dieser Gründung beigezogen wurde, dürfte als ein Zeichen seines damaligen Ansehens bei den schweizerischen Gelehrten zu werten sein. Ferner scheint mir interessant, daß Lutz von hinterlassenen Manuskripten wußte, denen nachzuspüren vielleicht lohnen würde. Endlich ist auch die genaue Angabe über den Aufenthalt Mumenthalers in Paris im Jahre 1773 bedeutsam. Da mir die von Lutz erwähnte Wochenzeitung L'Avant Coureur nicht gleich zugänglich war, suchte ich in den Publikationen der Académie Royale des Sciences in Paris aus den Jahren 1773 und 1774 nach der offiziellen Bestätigung für die erwähnte Prüfung und Anerkennung der Instrumente Mumenthalers, fand dort aber nicht den geringsten Hinweis, was mich eher skeptisch werden ließ.

Geht man noch einen Schritt weiter zurück, so stößt man in einem Rarissimum, dem ersten kantonal-bernischen Firmenadreßbuch von 1795 auf die einzige bisher bekannte Äußerung Mumenthalers über seine Tätigkeit. In dem genannten Büchlein sind neben Handelshäusern und Künstlern auch «ansehnliche gewerbtreibende Personen und Profeßionisten» verzeichnet. Jacob Mumenthaler hatte der Redaktion über sein Leben berichtet, und diese suchte seinen aus dem Rahmen der übrigen aufgezählten Geschäfte und Betriebe herausfallenden Ausführungen durch den Abdruck gerecht zu werden. Der Text im Berner Firmenadreßbuch von 1795 (S. 27-28) lautet: «Jakob Mumenthaler, ein sehr erfinderischer Kopf; sonst ein Buchbinder, treibt aber die Profession schon lange nicht mehr. Er schrieb uns in einem Briefe von sich folgende Lebensumstände:

Die Buchbinder-Profession, zu der ich in meiner Jugend gewidmet worden, hat mich mit Büchern bekannt gemacht, und hier zeigte sich meine Neigung und Lust zur Physik, und vorzüglich zur Verfertigung der optischen Maschinen. Da ich deswegen mit den Liebhabern derselben Bekanntschaft suchte, so reiste ich nach Lengnau zu Herrn Pfarrer Tribolet, der ein großer Liebhaber von physicalischen und vorzüglich optischen Maschinen 
war. Hier lernte ich das Branderische Sonnen-Microskop kennen, und verfertigte auch dergleichen.

Da die Electricität so großes Aufsehn zu machen anfing, so verfertigte ich auch elektrische Maschinen; und habe auch dergleichen selbst inventiert, darunter sich eine befindet, die von jedem Liebhaber der electr. Experimenten sollte angeschafft werden [folgt deren Beschreibung].

In meinem Cabinet (wenn ich es so nennen darf) sind dermalen vorhanden: Spiegel-Telescope, Latternen-Mag., Camera-obscura, Polihedron mit Figuren, Glas-Conus mit Figuren, Microscop-Solare, Microscop-Comp. et Simp., Prospecten-Maschinen, verschiedene sogenannte optische FeuerwerkMaschinen, darunter eine große mit Mahlereyen, Cylinder- und Conus-Spiegel mit Figuren, electr. Maschinen und Apparat, als: Scheiben- und Kugelmaschinen mit sehr viel Apparat zu Experimenten, auch der electr. Zunder oder die Lampe.» [Es folgen noch magnetische Instrumente, ferner Farben, Lacke und gegossene Kerzen aller Art.]

Auch in diesem Artikel finden sich mehrere bedeutsame Äußerungen. 1. ist Mumenthaler dem Berufe des Buchbinders «gewidmet worden », d.h. also, er hat ihn nicht so überlegt gewählt, wie es bei Lutz klang. 2. gibt er selbst an, Belehrung bei einem Pfarrer Tribolet ${ }^{1}$ in Lengnau gesucht und erhalten zu haben, und 3. lernen wir nun einige der von ihm angefertigten Apparate und Instrumente kennen, darunter die im 18. Jahrhundert bei Liebhabern der Naturbeobachtung so verbreiteten einfachen und zusammengesetzten Mikroskope, speziell auch das Brandersche Sonnen-Mikroskop ${ }^{2}$.

1 Über den Pfarrer Johann Jacoв Tribolet (1689-1761) weiß man nur wenig. Sein Zeitgenosse GRUNER schreibt in seinen Genealogien bernischer Geschlechter von ihm: Ein guter Literatus, Chymicus und in allen Scienzen wolerfahren, doch dabei seltsamen humors (Burgerbibliothek Bern, Mss. Hist. Helv. XVII. 54). In den Monatlichen Nachrichten einicher Merkwürdigkeiten vom Jahre 1762 (Zürich 1763) wird auf S. 26 sein Tod gemeldet; dabei heißt es: «Er war ein großer Liebhaber und Kenner der Naturalien, wie er dann eine schöne Sammlung darvon, wie auch von Mathematischen und sonst kostbaren Bücheren hinterlassen hat.»

2 Dieses Instrument ist eigentlich eine Art Mikroprojektionsapparat. Als intensivste aller damals bekannten Lichtquellen wurde dabei direktes Sonnenlicht verwendet, mit dem transparente Objekte durchstrahlt wurden. Maximale Bildhelligkeit war aber nur zu erreichen, wenn die optische Achse des Instrumentes ständig auf die Sonne gerichtet blieb. Brander löste bei seinem Mikroskop dieses Problem, indem er es als Ganzes sowohl in horizontaler wie in vertikaler Richtung verschiebbar machte. Damit dürfte es gegen Schwingungen ziemlich empfindlich gewesen sein, die natürlich die Bildschärfe verringerten. 
Unser Weg ist jedoch noch nicht zu Ende. Gehen wir weiter zurück, so stoßen wir auf eine noch ausgiebigere Quelle bei Hans Jаков Holzhalb, der ein Supplement zu einem allgemeinen helvetisch-eidsgenößischen oder schweizerischen Lexikon so von ... Hans Jakob Leu ... in alphabetischer Ordnung behandelt worden herausgab. Im 4. Teil (Zürich 1789) steht auf S. 277 das Wesentliche über den Mechanicus Mumenthaler, der als einer der «ersten die elektrischen Maschinen von dichtem und starken Papier erfunden hat, welche noch stärkeren Effekt als die Gläserne machen sollen; er verfertigte auch papierene Electrophoren, die besser sind als die von Pech; hat auch ein Microscopium von neuer Invention erfunden, welches im Journal Helvetic. 1774 im Jän. beschrieben worden.»

Hier scheint mir wichtig, daß Mumenthaler als gelernter Buchbinder seine Fertigkeit in der Handhabung von Papier von verschiedener Art auch für den Bau elektrischer Apparate ausnützte; ferner war der Hinweis auf eine Beschreibung des von Mumenthaler verbesserten Mikroskopes in einer schweizerischen Zeitschrift bedeutsam, zumal sie zeitlich ganz kurz auf dessen Pariser Aufenthalt folgte. Es lag daher nahe, einen Zusammenhang zwischen diesen beiden Ereignissen anzunehmen. Gerade als ich mit meinen Bemühungen um Jacob Mumenthaler bis zu diesem Punkte gelangt war, erhielt ich Kenntnis von dem Inhalt der mich interessierenden Nachrichten aus der Wochenschrift L'Avant Coureur von 1773. Meine Bitte nachzuprüfen, ob der betreffende Jahrgang vollständig in einer Pariser Bibliothek vorhanden sei, beantwortete Herr Dr. J. Schiller, Paris, in außerordentlich dankenswerter Weise durch die Übersendung einer eigenhändigen Abschrift aller darin enthaltenen auf Mumenthaler bezüglicher Nachrichten. So war ich in der Lage, die angeblich offizielle Anerkennung der Instrumente Mumenthalers durch die Pariser Académie des Sciences überprüfen zu können und die im Avant Coureur vom 27. September und 11. Oktober 1773 vorhandenen Beschreibungen seines Mikroskopes mit der im Januar 1774 erfolgten Publikation im Nouveau Journal Helvétique zu vergleichen.

L'Avant Coureur enthält zwei in ihrer Art verschiedene, sachlich jedoch zusammengehörige Mitteilungen. Die erste ist eine redaktionelle Notiz, die möglicherweise durch Mumenthaler selbst veranlaßt wurde. Sie lautet: «Optique. Le microscope solaire inventé par Lieberkuhn, et corrigé par les Anglais, ne peut servir, comme les Amateurs le savent, que pour de petits objets transparens. Plusieurs savants se sont donné la peine de remédier à ce défaut. Mais comme ils n'avoient pas d'ouvriers assez habiles, on a été obligé de s'en contenter. 
Un amateur de la science optique, Suisse de nation, nommé Jacques Mumenthaler, qui possède la théorie et la pratique, a enfin remédié à ce défaut, et mis cet instrument en état de servir pour tous les objets opaques, de manière qu'on peut en voir dans la chambre obscure, très-distinctement et grossit toutes les pièces de monnoyes et médailles, toutes sortes de petits tableaux, portraits et tailles douces, toutes sortes de graines et fleurs naturelles, des coquillages, des insectes, etc. Cet artiste a apporté de ces instruments à Paris. Il en fera l'épreuve, devant les amateurs qui voudront en acheter, dans son logis ou dans les leurs, s'ils ont une chambre qu'il puisse obscurcir et arranger pour cela.

Il loge rue des 4 vents, dans le cul-de-sac chez M. Girardot. Il ne peut s'arrêter à Paris que peu de jours " (L'Avant Coureur, 1773, No 39,27 septembre, p. 611-612).

Die zweite Mitteilung (gleicher Jahrgang, Nr. 41, 11. Oktober, S. 645648 ) ist ein an den Herausgeber gerichteter Brief. Dieser ist in den wesentlichen Teilen identisch mit der Publikation im Nouveau Journal Helvétique (Januar 1774); auf kleine Abweichungen dieser Veröffentlichung gegenüber der in Paris erschienenen Mitteilung wird in den Anmerkungen verwiesen. Im Avant Coureur (1.c.) heißt es: «Lettre de Mr. De Bernieres ${ }^{3}$, écuyer, l'un des quatre contrôleurs-généraux des ponts et chaussées en France, de la société royale des sciences de Metz, et de l'académie royale des belles-lettres de Caen, en date du 27 septembre 1773, à l'auteur de L'Avant Coureur sur le nouveau microscope solaire de M. Mumenthaler.

\section{Monsieur}

Je me hâte de vous informer de l'invention d'un nouveau microscope solaire, dont les effets doivent intéresser les curieux. Le nommé Jacques Mumenthaler, Suisse de nation, vient de me faire voir un de ses microscopes. Il en a apporté un grand nombre de son pays. Les artistes qui ont pris le nom et le fond du feu sieur Passement, en ont acheté plusieurs. Ce sont des marchands fort honnêtes qui se contentent d'un gain modique: ils sont aussi très en état d'en faire de pareils, et même d'y ajouter de nouvelles perfec-

${ }^{3}$ Bernieres (geboren zu Anfang des 18. Jahrhunderts, gestorben 1783) war von Beruf Ingenieur. Er ist bekannt als Erfinder hydraulischer Maschinen. Daß er sich auch mit der theoretischen Optik befaßte zeigt seine Schrift Abrégé des propriétés des miroirs concaves, 1760 (Grande Encyclopédie VII, p. 389). 
tions. Ils nous flattent en outre de nous les procurer à un prix plus avantageux que leur inventeur a exigé ${ }^{4}$.

Ces microscopes réunissent plusieurs avantages qu'on netrouve pas dans les autres. Ce n'est point par des cordes, passant par des poulies, que le miroir extérieur tourne pour prendre les rayons du Soleil, et les ramener horizontalement dans la chambre; c'est par le mouvement de deux roues dentées de cuivre, qui s'engrènent. La base de ces microscopes est une plaque de cuivre poli qu'on attache à une fenêtre par les quatre coins avec des vis, et sur le milieu de laquelle se visse le principal tuyau. Le miroir extérieur s'incline plus ou moins, selon le besoin, par le moyen d'une vis qui le pousse en avant quand on la tourne à droite, et qui le laisse revenir sur lui-même par son propre poids quand on la tourne à gauche.

On obtient surtout deux effets singuliers de cette sorte de microscope; c'est $1^{\circ}$ de voir en-dedans de la chambre, sur un carton blanc vertical, l'image très-grossi et très-nette des objets opaques collés ${ }^{5}$ à la thérébentine sur de petits porte-objets qu'on place dans une coupure pratiquée transversalement à l'un des tuyaux de rechange du microscope, à la distance du foyer de sa petite lentille. Il y a en-dedans de ce tuyau un petit miroir plan de métal, qu'on incline plus ou moins, au moyen d'une vis dont la tête sort par le bout de ce tuyau, pour faire tomber la lumière sur le porte-objet, qui dans ses différents cases contient des graines, des sables, de petits coquillages, etc.

$2^{\circ}$ On ôte ce tuyau de dedans le tuyau principal, et on met en sa place une petite boîte carrée à peu près; mais qui se termine en avant par un tuyau qui entre dans celui par où arrivent les rayons de lumière. Il y a dans le fond ultérieur de cette boîte un miroir incliné ${ }^{6}$, de manière qu'il renvoie la lumière vers le fond opposé de cette boîte, et au-dessus du tuyau par où cette lumière arrive. Ce fond est ouvert en cet endroit, et l'on y appuye verticalement et renversés de petites estampes ou de portraits en miniature de la grandeur au plus d'un écu de six livres. Ces estampes ainsi placées tout près de cette ouverture, se trouvent fort éclairées par le reflet du miroir incliné

4 Nouveau Journal Helvétique, 1774, p. 31 : «Le nommé Jacques Mumenthaler, Suisse de nation, a inventé depuis peu un nouveau microscope très curieux; il en a porté un grand nombre à Paris, où il les vend cinq louis avec tous les accessoires.»

5 Nouveau Journal Helvétique, 1774, p. 32 : Statt «celles à la thérébentine» muß es «collés à la thérébentine» heißen.

${ }^{6}$ Zusatz im Nouveau Journal Helvétique, 1774, p. 33 : «de glace etamée». 
dont nous venons de parler, et à la distance convenable d'une loupe de cinq pouces de foyer, qui termine la boîte du coté du fond de la chambre. Leur lumière, passant à travers cette loupe, va porter leur image à peu-près de grandeur naturelle, sur le carton blanc, à six ou huite pieds de distance, et avec beaucoup plus de netteté et de perfection qu'on ne le voit par le lanterne magique. Si on met à la place de ces miniatures une pièce de monnaie, un louis d'or, par exemple, il paroîtra sous un pied de diamètre au plus ${ }^{7}$; si on y met une montre, son cadran semblera être celui d'une grande pendule; si on y met des fleurs collées à plat sur une carte, elles iront se peindre aussi très-grosses sur le carton, et avec toutes leurs couleurs, etc.

Ce microscope produit, au surplus, tous les autres effets qu'on obtient du microscope solaire connu, et m'a paru plus solidement exécuté, et plus commode que tout ce qu'on a vu jusqu'à présent en ce genre. Les amateurs doivent désirer d'en avoir dans leurs cabinets; l'Histoire Naturelle et la physique ne peuvent qu'y gagner: c'est l'objet de ma lettre.

J'ai l'honneur d'être, etc.»

Das ist also der Inhalt der beiden Publikationen über das von Jacob Mumenthaler verbesserte Sonnenmikroskop. Aus der Mitteilung im Avant Coureur geht hervor, daß nicht die Académie des Sciences in Paris seine Instrumente prüfte, sondern ein Ingenieur, der Mitglied der Naturforschenden Gesellschaft in Metz und der Akademie der schönen Künste in Caen war; immerhin war BERNIERES auf optischem Gebiet erfahren (vgl. Anm. 3 ). Daß die von Mumenthaler konstruierten Mikroskope solider waren als das ihm zum Vorbild dienende Brandersche Sonnenmikroskop, ist leicht verständlich; er hatte auf die direkte Richtung des Instrumentes gegen die Sonne verzichtet und dafür außen am Fenster einen kipp- und neigbaren Spiegel angebracht, dessen Stellung durch Zahnräder bzw. eine Schraube vom Zimmer aus regulierbar war. So erhielt er horizontalen Lichteinfall und konnte das ganze Instrument in dieser Stellung fest montieren. Als weitere Neuerung sei auf den geneigten Spiegel im Innern des Kastens verwiesen, durch den er bei undurchsichtigen Objekten eine intensive Auflicht-Beleuchtung erzielte. Diese Lösung, auch undurchsichtige Teile im Sonnenmikroskop untersuchen zu können, wird bisher Martin zugeschrieben. Angeblich stammt seine Erfindung aus dem Jahre 1770 (CLAY und Court, S. 222); seine Publikation erschien jedoch erst 1774. Es kann nicht bezweifelt werden, daß Mumenthaler die gleiche Lösung selbständig und mindestens

7 Im Nouveau Journal Helvétique, 1774, p. 34 steht «et plus» statt «au plus». 
gleichzeitig fand, denn wenn er 1773 schon eine größere Zahl von Mikroskopen dieser Art in Paris zum Verkauf bringen konnte, so muß er doch die Entwicklungsarbeiten einige Zeit früher ausgeführt haben. Auch die Anfertigung der zum Verkauf bestimmten Mikroskope erforderte Zeit. Ihre Beschreibung im Avant Coureur (September-Oktober 1773) und im Nouveau Journal Helvétique (Januar 1774) nannte seinen Namen als den des Erfinders der Neuerungen. Zeitlich gehen diese Publikationen der von Martin voraus.

Leider kann ich bisher keine Abbildung eines der von Mumenthaler gebauten Sonnenmikroskope vorlegen. Trotz mancher Bemühungen habe ich in schweizerischen Museen und Sammlungen noch kein derartiges Instrument gefunden. Meine Hoffnung ist noch Paris, wo sich vielleicht eines der damals verkauften Instrumente erhalten haben könnte. Indessen geht meine Bitte an alle Leser dahin, bei gelegentlichem Besuch in Museen und Sammlungen auf solch ein Instrument zu achten, das an der Grundplatte aus poliertem Kupfer kenntlich ist und dessen Spiegel durch zwei ineinander greifende Zahnräder und eine Schraube bewegt werden kann.

Als einen Nebenbefund erwähne ich noch, daß ich bei der Suche nach Instrumenten aus Mumenthalers Werkstatt mehrere sogenannte Nürnberger Mikroskope gesehen habe bzw. ermitteln konnte, die auf der Untereite der hölzernen Basisplatte die Buchstaben IM in einem querovalen Ring eingebrannt zeigen. Bezeichnend ist der Punkt über dem I, er soll wohl verhindern, die Marke bei Drehung um $180^{\circ}$ als WI zu lesen. Ich selbst habe zwei solcher Instrumente in Händen, die Zürcher medizinhistorische Sammlung besitzt eines und das Basler Pharmaziehistorische Museum deren zwei. Es scheint danach, als gäbe es eine gewisse Häufung dieser İM signierten Mikroskope in der deutschsprachigen Schweiz, was mit einiger Wahrscheinlicckeit daran denken ließe, daß sie auf Jacob Mumenthaler zurückgehen. Auch diesbezüglich wäre mir die Aufmerksamkeit jedes Lesers erwünscht, denn jede Meldung positiver wie negativer Natur würde eine Hilfe zur weiteren Klärung der Tätigkeit von Jacob Mumenthaler bedeuten.

Frau Dr. M. Rooseвоom, Leiden, erteilte mir freundlicherweise brieflich die Auskunft, daß die Signatur IM bei Mikroskopen des sogenannten «Nürnberger» Typus nicht selten ist; sie nannte dafür auch einige Möglichkeiten der Deutung und wies auf Unterschiede in der Umrahmung hin. Ihre Angaben beziehen sich allerdings nicht auf die hier besonders betonte Signatur IM (mit dem Punkt über dem I). 


\section{Literatur}

Clay Reginald S. und Thomas H. Count, The History of the Microscope, London 1932. Feller Richard, Geschichte Berns, vol. 3, Bern 1955.

Harting P., Das Mikroskop, deutsche Originalausgabe, Braunschweig 1859.

HolzhalB HaNs $\mathrm{J}_{\mathrm{AKOB}}$, Supplement zu einem allgemeinen helvetisch-eidsgenößischen oder schweizerischen Lexikon so von ... Hans Jakob Leu ... in alphabetischer Ordnung behandelt worden, 4. Theil, Zürich 1789.

KoHLEr Eugen, Alt-Langenthal in Wort und Bild, Langenthal 1932.

Lutz Markus, Moderne Biographien, Vol. 2, Lichtensteig 1826.

SiEgrried J., Geschichte der Schweizerischen Naturforschenden Gesellschaft ..., Zürich 1865.

Tribolet H., Artikel «Mumenthaler» in Historisch-Biographisches Lexikon der Schweiz, 5. Band, Neuenburg 1929.

- Sammlung bernischer Biographien, 5. Band, Bern 1906.

- L'Avant Coureur, Paris 1773.

- Berner Addreß-Handbuch der Handelshäuser ... wie auch der Künstler, ansehnlichen gewerbtreibenden Personen und Profeßionisten, Bern 1795.

- Nouveau Journal Helvétique, Neuchâtel 1774. 